\title{
VICTOR MATVEEVICH BUCHSTABER'S 70TH BIRTHDAY ANNIVERSARY
}

April 1, 2013 marked the 70th birthday anniversary of Victor Matveevich Buchstaber, a mathematician with multifarious interests and a wonderful person, a Corresponding Member of the Russian Academy of Sciences.

Victor Matveevich Buchstaber is the author of fundamental contributions to algebraic topology, theory of Abelian functions, mathematical physics, and applied statistics. His papers are full of ideas, many of which served as a basis of new active areas in mathematics. Buchstaber's achievements are well known and recognized everywhere in the world.

He is the Vice-President of the Moscow Mathematical Society, a member of the National Committee of Mathematicians of Russian Federation, a Foreign Member of the

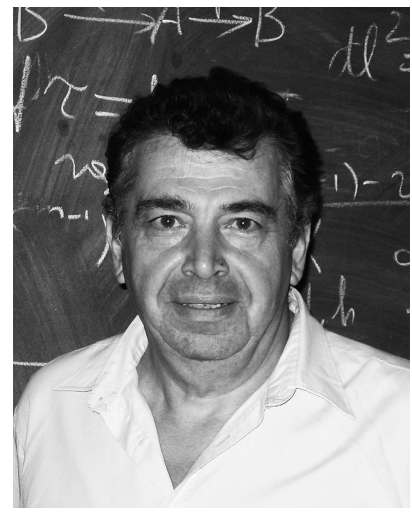
Edinburgh Mathematical Society (Academy of Sciences of Scotland) and an Honorary Professor of the University of Manchester. He was an invited speaker at the International Congress of Mathematicians in Vancouver (1974), gave lectures at many leading mathematical centers around the world, and was the Adams Memorial Lecturer at the J. Adams Memorial Conference in Manchester (1995).

Buchstaber is the author of more than 250 publications, including five monographs. Among his students are two doctors of science and more than 20 candidates of science. Results of Buchstaber's scientific school are widely recognized.

On June 18-22, 2013 the Steklov Mathematical Institute of the Russian Academy of Sciences hosted the International Conference on Algebraic Topology and Abelian Functions devoted to Victor Matveevich Buchstaber's 70th birthday anniversary. More than 150 mathematicians from more than 20 countries participated in the conference. This issue is the first volume of the proceedings of this conference; the second volume will be published in 2014-2015 in the Proceedings of the Steklov Mathematical Institute.

A. A. Gaifullin

T. E. Panov 